\title{
Lymphovascular invasion as a negative prognostic factor for triple-negative breast cancer after surgery
}

\author{
Ki Jung Ahn, MD, PhD ${ }^{1^{*}}$, Jisun Park, $\mathrm{MD}^{2^{*}}$, Yunseon Choi, MD, PhD ${ }^{1}$ \\ Departments of ${ }^{1}$ Radiation Oncology and ${ }^{2}$ Nuclear Medicine, Inje University Busan Paik Hospital, Busan, Korea
}

\begin{abstract}
Purpose: This study aimed to evaluate the prognostic effects of lymphovascular invasion (LVI) in triple-negative breast cancer (TNBC) patients who underwent surgical resection.

Materials and Methods: A total of 63 non-metastatic TNBC patients who underwent surgical resection were retrospectively investigated from 2007 to 2016 in Inje University Busan Paik Hospital. Pathological tests revealed that 12 patients (19.0\%) had LVI. Approximately $61.9 \%(n=39)$ of the patients' samples stained positive for p53. Additional chemotherapy and radiotherapy (RT) were performed in $53(84.1 \%)$ and $47(74.6 \%)$ patients, respectively.

Results: The median follow-up period was 39.5 months (range, 5.9 to 123.0 months). The pathological T stage ( $p=0.008)$, N stage $(p=0.014)$, and p53 positivity $(p=0.044)$ were associated with LVI. Overall, the 3 -year disease-free survival (DFS) rate and overall survival (OS) rate were $85.4 \%$ and $90.2 \%$, respectively. Ten patients (15.9\%) experienced relapse. LVI $(n=12)$ was associated with relapses $(p=0.016)$. $p 53$ positivity was correlated with poor DFS $(p=0.048)$. Furthermore, LVI was related to poor DFS $(p=0.011)$ and OS ( $p=0.001)$ and considered as an independent prognostic factor for DFS $(p=0.039)$. The 3-year DFS of patients with LVI (n $=12$ ) was only $58.3 \%$. Adjuvant RT minimized the negative prognostic effect of LVI on DFS ( $p=0.068$ [with RT] vs. $p=0.011$ [without $\mathrm{RT}]$ ).

Conclusion: LVI was related to the detrimental effects of disease progression and survival of TNBC patients. Thus, a more effective treatment strategy is needed for TNBC patients with LVI.
\end{abstract}

Keywords: Lymphovascular invasion, Triple negative breast neoplasms, Survival, Prognosis, Radiotherapy

\section{Introduction}

Lymphovascular invasion (LVI) has been a topic of interest since it is currently considered as an important prognostic factor for primary breast cancer $[1,2]$. Schoppmann et al. [3] previously reported that LVI is an independent prognostic factor for disease-free survival (DFS) and overall survival (OS) of patients with breast cancer. Moreover, LVI was associated with the development of lymph node metastases and lymphatic microvessel density [3]. Another clinical study conducted by Krishnamurti et al. [4] showed that LVI may be associated with peripheral tumor-infiltrating lymphocytes, which have a clinical significance in breast cancer [5]. Although controversial [6], LVI may have a prognostic significance in patients with triple-negative breast cancer (TNBC) [7], which has a poor prognosis compared to other intrinsic subtypes of

Received 17 August 2017, Revised 13 September 2017, Accepted 10 October 2017.

Correspondence: Yunseon Choi, MD, PhD, Department of Radiation Oncology, Inje University Busan Paik Hospital, 75 Bokji-ro, Busanjin-gu, Busan 47392, Korea. Tel: +82-51-890-8606, Fax: +82-51-891-1754, E-mail:ttyoon@gmail.com

*Both authors contributed equally to this work.

(c) This is an Open Access article distributed under the terms of the Creative Commons Attribution Non-Commercial License (http://creativecommons.org/ licenses/by-nc/4.0/) which permits unrestricted non-commercial use, distribution, and reproduction in any medium, provided the original work is properly cited.

www.e-roj.org 
breast cancers [8,9]. A recent study by Gujam et al. [10] showed that breast cancer subtypes could affect the outcomes related to LVI. The prognosis of hormone receptor-negative breast cancers, such as TNBC, is suggested to be highly influenced by LVI compared to that of hormone receptor-positive breast cancers [10].

Therefore, we assumed that TNBC patients with LVI would have a worse prognosis than those without LVI based on pathological reports. This study investigated the effects of LVI with a focus on the poor prognosis of patients with TNBC after surgical resection. Because the prognostic significance of LVI was underestimated till recently, this study aimed to evaluate the prognostic effects of LVI in TNBC patients who underwent resection.

\section{Material and Methods}

\section{Patients}

This retrospective study included 63 non-metastatic TNBC patients who underwent surgical resection. Patient records from the electronic medical records and imaging studies were reviewed from February 2007 to November 2016 at Inje University Busan Paik Hospital. Breast cancer staging was based on the 7th edition of the American Joint Committee on Cancer (AJCC) Manual. Pathological T1-2NO-3MO TNBC patients were evaluated in this study.

Table 1 illustrates the characteristics of the evaluated patients. Based on the T stage, T1 and T2 accounted for 60.3\% $(n=38)$ and $39.7 \%(n=25)$ of the patients, respectively. In addition, one-third ( $n=21,33.3 \%$ ) of the patients had regional lymph node metastases based on pathological reports. A detailed pathologic node stage of the patients included the following: 42 (66.7\%) with N0, 16 (25.4\%) with N1, 3 (4.8\%) with N2, and 2 (3.2\%) with N3.

All patients evaluated in this study were women who underwent curative surgical resection for invasive breast cancer. Breast-conserving surgery (BCS) was performed in $49(77.8 \%)$ patients. The remaining 14 (22.2\%) patients underwent modified radical mastectomy (MRM). Most patients with advanced-stage breast cancer received chemotherapy, which was performed in $84.1 \%(n=53)$ of the patients with standard treatment regimens, of which 6 received neoadjuvant chemotherapy before surgical resection. Anthracycline- and taxane-based regimens were commonly used in chemotherapy [11]. Most patients who underwent BCS received adjuvant RT. Generally, patients with locally advanced-stage breast cancer who were treated with MRM underwent radiotherapy (RT)
Table 1. Patients' characteristics

\begin{tabular}{|c|c|}
\hline Characteristic & No. $(\%)$ \\
\hline \multicolumn{2}{|l|}{ Age (yr) } \\
\hline$<50$ & $22(34.9)$ \\
\hline$\geq 50$ & $41(65.1)$ \\
\hline \multicolumn{2}{|l|}{ p53 } \\
\hline Positive & $39(61.9)$ \\
\hline Negative & $24(38.1)$ \\
\hline \multicolumn{2}{|l|}{ Resection margin } \\
\hline Positive & $1(1.6)$ \\
\hline Negative & $62(98.4)$ \\
\hline \multicolumn{2}{|l|}{ Perineural invasion } \\
\hline Positive & $3(4.8)$ \\
\hline Negative & $60(95.2)$ \\
\hline \multicolumn{2}{|l|}{ Lymphovascular invasion } \\
\hline Positive & $12(19.0)$ \\
\hline Negative & $51(81.0)$ \\
\hline \multicolumn{2}{|l|}{ pathologic T stage } \\
\hline $\mathrm{T} 1$ & $38(60.3)$ \\
\hline $\mathrm{T} 2$ & $25(39.7)$ \\
\hline \multicolumn{2}{|l|}{ pathologic N stage } \\
\hline No & $42(66.7)$ \\
\hline N1 & $16(25.4)$ \\
\hline N2 & $3(4.8)$ \\
\hline N3 & $2(3.2)$ \\
\hline \multicolumn{2}{|l|}{ Tumor laterality } \\
\hline Right & $33(52.4)$ \\
\hline Left & $30(47.6)$ \\
\hline \multicolumn{2}{|l|}{ Type of surgery } \\
\hline BCS & $49(77.8)$ \\
\hline MRM & $14(22.2)$ \\
\hline \multicolumn{2}{|l|}{ Radiotherapy } \\
\hline Yes & $47(74.6)$ \\
\hline No & $16(25.4)$ \\
\hline \multicolumn{2}{|l|}{ Chemotherapy } \\
\hline Yes & $53(84.1)$ \\
\hline No & $10(15.9)$ \\
\hline
\end{tabular}

BCS, breast-conserving surgery; MRM, modified radical mastectomy.

when large primary tumors or lymph node metastases are observed (axilla, supraclavicular area, or internal mammary chain). Forty-seven patients received RT, of which 43 were treated with RT after BCS and 4 were treated with RT after mastectomy. Among the patients who underwent RT after BCS $(n=43), 10$ received supraclavicular lymph node irradiation. RT was performed in patients with current standard treatment regimens. The median total RT dose was $60.4 \mathrm{~Gy}$ (range, 50 to $66 \mathrm{~Gy}$ ). Whole-breast RT was generally performed using 50-54 Gy with photon and tangential field. Next, patients who 
underwent BCS were treated with an electron boost dose to the tumor bed at 9-16 Gy. In RT cases, all patients were treated with conventional fraction RT (1.8-2 Gy per fraction).

\section{Pathological examination}

TNBC was defined as the lack of hormone receptor expression (negative for both estrogen and progesterone receptors) and human epidermal growth factor receptor 2 (HER2; expression score $0-1$ or 2 (negative) with fluorescence in situ hybridization test) by immunostaining. The antibodies used in this study are as follows: estrogen receptor (1:90; Novocastra Laboratories Ltd., Newcastle upon Tyne, UK), progesterone receptor (1:170; Novocastra Laboratories Ltd.), and HER2 (Ventana Medical Systems Inc., Tucson, AZ, USA). p53 positivity was measured via immunostaining with p53 antibody (1:150; Dako, Glostrup, Denmark).

\section{Statistical analysis}

SPSS software ver. 18.0 (SPSS Inc., Chicago, IL, USA) was used for statistical analyses. The primary end-point of this study was DFS. The secondary end-points were the rate of the overall relapse, locoregional recurrence, distant metastasis, and OS. Locoregional recurrence was defined as the reappearance of tumor in the ipsilateral breast/chest wall and/or ipsilateral regional lymph nodes. Distant metastasis was defined as tumor spread to distant organs or lymph nodes.

The Fisher exact test was used in evaluating the clinical factors related to LVI and recurrence. The Kaplan-Meier method was utilized for the univariate analysis of survival. The log-rank test was used in finding the prognostic factors. The Cox-regression test was utilized for the multivariate analysis of survival. A multivariate analysis of the statistically significant factors $(p<0.05)$ in a univariate analysis was performed using the Cox model.

\section{Results}

\section{Patients}

The median follow-up period and age of the patients were 39.5 months (range, 5.9 to 123.0 months) and 54 years (range, 30 to 77 years), respectively. Table 1 shows the patient and treatment characteristics. Pathologically, a total of 39 (61.9\%) patient samples were $\mathrm{p53}$ positive on immunostaining and LVI was observed in $19.0 \%(n=12)$ of the patients. Only one $(1.6 \%)$ patient had a positive resection margin after surgical resection. Perineural invasion was observed in $3(4.8 \%)$ patients based on the pathological reports. Postoperative RT was carried out in
Table 2. Comparison according to lymphovascular invasion

\begin{tabular}{lccc}
\hline \multirow{2}{*}{ Characteristic } & \multicolumn{2}{c}{ Lymphovascular invasion } & \\
\cline { 2 - 3 } & $\begin{array}{c}\text { Negative } \\
(\mathrm{n}=51)\end{array}$ & $\begin{array}{c}\text { Positive } \\
(\mathrm{n}=12)\end{array}$ & p-value \\
\hline Age $(\mathrm{yr})$ & 16 & 6 & 0.314 \\
$<50$ & 35 & 6 & \\
$\geq 50$ & & & 0.044 \\
p53 & 35 & 4 & \\
Positive & 16 & 8 & \\
Negative & & & \\
pathologic T stage & 35 & 3 & \\
T1 & 16 & 9 & 0.008 \\
T2 & & & \\
pathologic N stage & 38 & 4 & \\
N0 & 13 & 8 & \\
N1-3 & & & \\
\hline
\end{tabular}

$47(74.6 \%)$ patients.

With regard to the clinical factors related to the LVI status (Table 2), the pathological T stage $(p=0.008), N$ metastases $(p=0.014)$, and $p 53$ positivity $(p=0.044)$ were significantly related to $L V I$ incidence. To be specific, LVI presented more frequently in higher $\mathrm{T}$ stage group compared to lower $\mathrm{T}$ stage group (T2 vs. T1; $p=0.008$ ). The presence of LVI was affected by the existence of lymph node metastases ( $p=0.014)$. In addition, p53 positivity was associated with a lower rate of LVI $(p=0.044)$.

\section{Pattern of failure}

During the follow-up period, 10 patients experienced relapse. Locoregional recurrence and distant metastases were observed in six and seven patients, respectively (three patients experienced both simultaneously). The most common distant metastatic site was the lungs.

LVI $(n=12)$ was related to relapse $(p=0.016)$ after surgery, which was more associated with distant metastases $(p=0.021)$ than locoregional recurrence $(p=0.077)$. Based on the analysis of the subgroup who underwent RT $(n=47)$, the overall relapse tended to be influenced by LVI $(p=0.075)$.

Among the 12 patients with $L V I$, those who received RT had a lower locoregional relapse rate $(22.2 \%, 2 / 9)$ than those who did not receive RT $(33.3 \%, 1 / 3)$. The patients who were treated with RT also had a lower distant metastasis rate than those who were not treated with RT (22.2\% [2/9] vs. 33.3\% [1/3]).

\section{Survival analysis}

The 3-year DFS rate and OS rate were 85.4\% and 90.2\% (Fig. 1), 


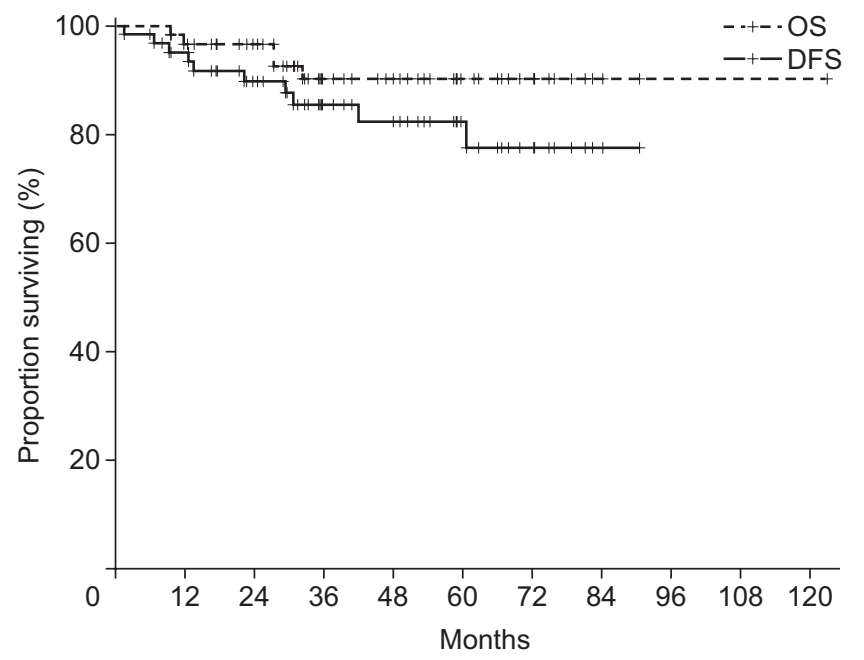

Fig. 1. Overall survival (OS) and disease-free survival (DFS) rates.

respectively. Additionally, the 3-year locoregional recurrencefree survival (LRRFS) and distant metastasis-free survival (DMFS) were 92.3\% and 89.4\%, respectively.

The univariate analysis showed that DFS was influenced by p53 positivity ( $p=0.048)$ and LVI ( $p=0.011)$ (Fig. 2, Table 3) OS was also significantly affected by LVI ( $p=0.001)$.

Based on the multivariable analysis of DFS (Table 4), LVI was considered an independent prognostic factor $(p=0.039)$. In addition, LVI was also considered as an independent prognostic factor based on the multivariable analysis of OS ( $p=0.029$ ) (Table 5). To be specific, the LRRFS was tended to be affected by the presence of LVI ( $p=0.057)$. The 3 -year cumulative LRRFS was $72.2 \%$ in patients with LVI and $97.5 \%$ in those without LVI. As for the DMFS, our study showed a significant difference between patients with LVI and those without LVI ( $p$ $=0.008$ ). In detail, the 3 -year cumulative DMFS was $66.7 \%$ in patients with LVI and $95.1 \%$ in those without LVI.

The influence of RT on DFS was also evaluated. Fig. 3 showed the comparison of DFS according to RT in the patient subgroup with LVI $(n=12)$. Although it was not extended to statistical significance because of its small sample size, the 3-year DFS of the patients who were treated with RT was higher compared to that of patients who did not undergo RT (66.6\% vs. 33.3\%; $p=0.376$ ) in the subgroup with LVI. According to another subgroup analysis, RT could reduce the

Table 3. Univariate analysis for disease-free survival and overall survival

\begin{tabular}{|c|c|c|c|c|c|}
\hline & No. of patients & 3-yr DFS (\%) & $p$-value & $3-y r$ OS (\%) & p-value \\
\hline Age (yr) & & & 0.354 & & 0.904 \\
\hline$<50$ & 22 & 79.9 & & 90.2 & \\
\hline$\geq 50$ & 41 & 88.7 & & 90.3 & \\
\hline p53 & & & 0.048 & & 0.066 \\
\hline Positive & 39 & 94.1 & & 97.2 & \\
\hline Negative & 24 & 72.9 & & 80.0 & \\
\hline Lymphovascular invasion & & & 0.011 & & 0.001 \\
\hline Positive & 12 & 58.3 & & 64.3 & \\
\hline Negative & 51 & 92.7 & & 97.9 & \\
\hline pathologic T stage & & & 0.052 & & 0.065 \\
\hline $\mathrm{T} 1$ & 38 & 90.5 & & 97.3 & \\
\hline $\mathrm{T} 2$ & 25 & 77.9 & & 80.2 & \\
\hline pathologic N stage & & & 0.792 & & 0.255 \\
\hline No & 42 & 84.8 & & 94.2 & \\
\hline $\mathrm{N} 1-3$ & 21 & 85.7 & & 83.2 & \\
\hline Type of surgery & & & 0.561 & & 0.045 \\
\hline BCS & 49 & 87.6 & & 94.7 & \\
\hline MRM & 14 & 77.4 & & 75.5 & \\
\hline Radiotherapy & & & 0.21 & & 0.451 \\
\hline Yes & 47 & 88.0 & & 92.3 & \\
\hline No & 16 & 78.0 & & 83.3 & \\
\hline Chemotherapy & & & 0.18 & & 0.341 \\
\hline Yes & 53 & 82.9 & & 88.5 & \\
\hline No & 10 & 100 & & 100 & \\
\hline
\end{tabular}

DFS, disease-free survival; OS, overall survival; BCS, breast-conserving surgery; MRM, modified radical mastectomy. 
Table 4. Multivariate analysis for disease-free survival

\begin{tabular}{lcc}
\hline & $H R(95 \% \mathrm{Cl})$ & $\mathrm{p}$-value \\
\hline Lymphovascular invasion & $3.75(1.07-13.14)$ & 0.039 \\
p53 & $0.33(0.08-1.29)$ & 0.112 \\
\hline
\end{tabular}

$\mathrm{HR}$, hazard ratio; $\mathrm{Cl}$, confidence interval.

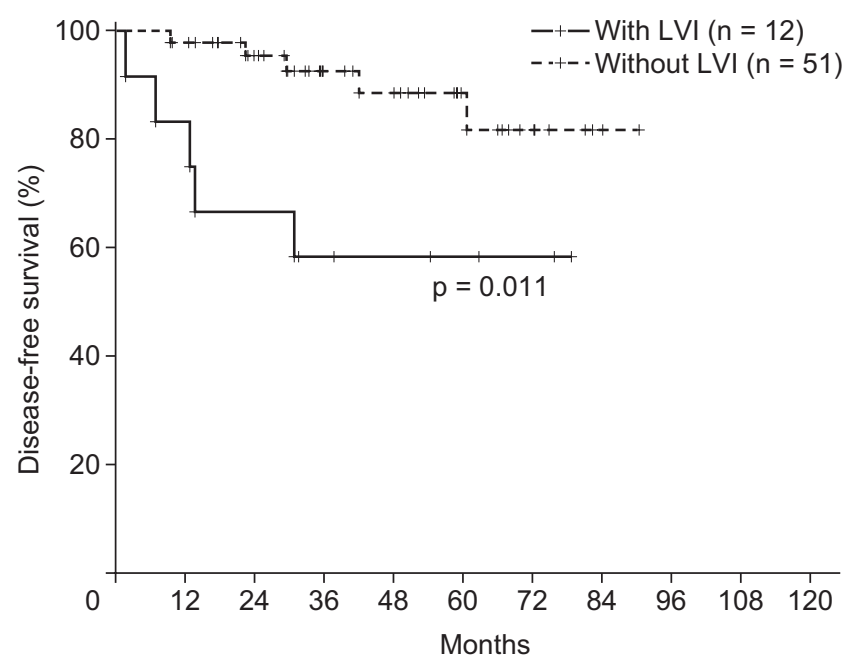

Fig. 2. Disease-free survival according to lymphovascular invasion $(\mathrm{LVI})$ in all patients.

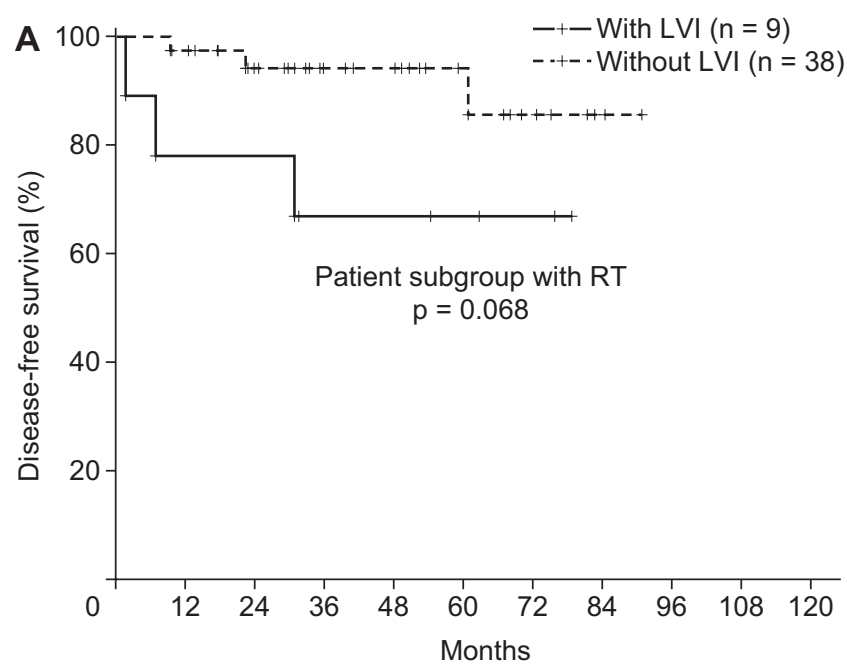

Table 5. Multivariate analysis for overall surviva

\begin{tabular}{lcc}
\hline & HR $(95 \% \mathrm{Cl})$ & p-value \\
\hline Lymphovascular invasion & $12.02(1.29-112.16)$ & 0.029 \\
Surgery type (BCS vs. MRM) & $0.32(0.05-1.99)$ & 0.222 \\
\hline
\end{tabular}

$\mathrm{HR}$, hazard ratio; $\mathrm{Cl}$, confidence interval; $\mathrm{BCS}$, breast-conserving surgery; MRM, modified radical mastectomy.

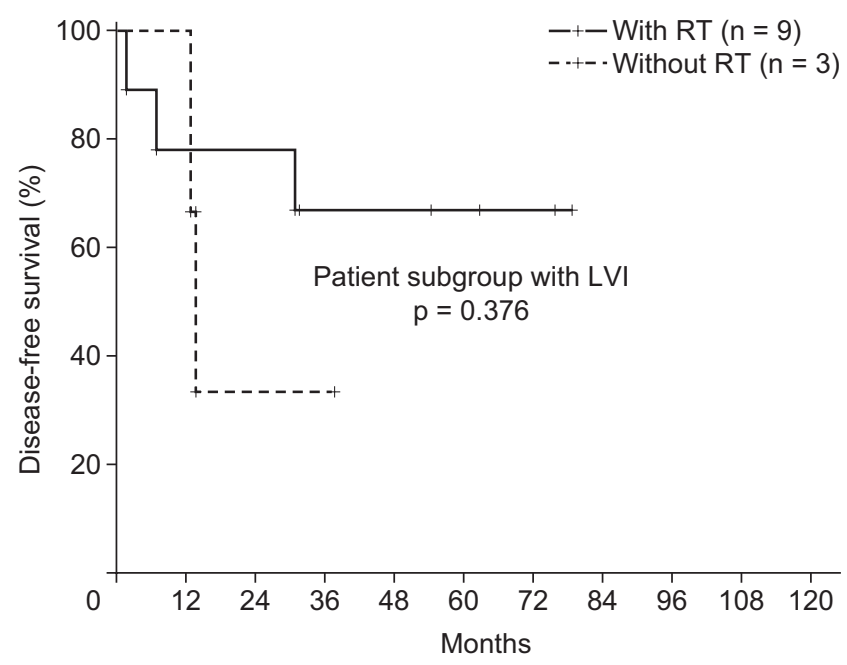

Fig. 3. Disease-free survival according to radiotherapy in the patients with lymphovascular invasion (LVI).

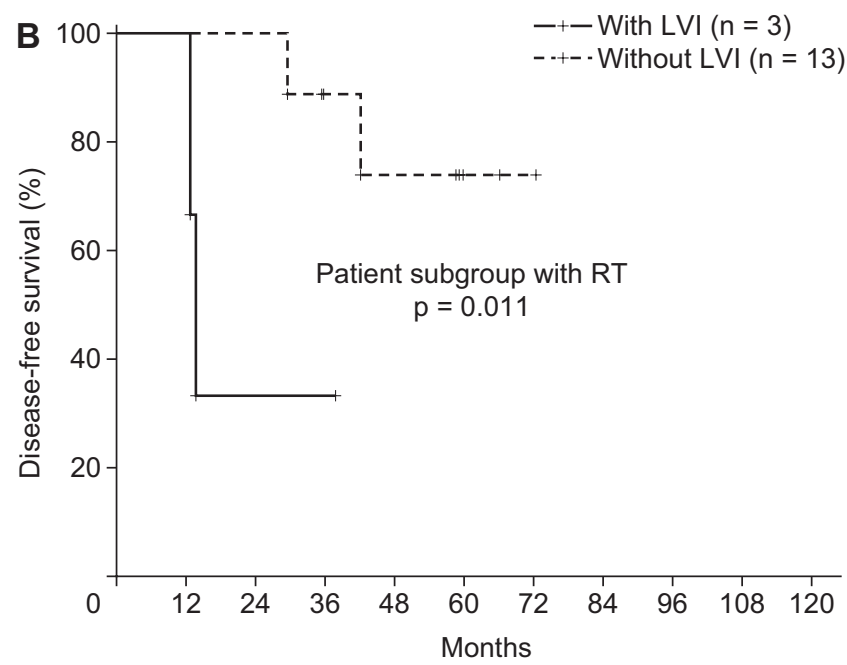

Fig. 4. Disease-free survival in the patients treated with adjuvant radiotherapy $(A)$ and the patients treated without radiotherapy (B) according to lymphovascular invasion (LVI).

negative effect of LVI on the prognosis of DFS ( $p=0.068$ [with RT] vs. $p=0.011$ [without RT]) (Fig. 4A and 4B). However, the effects of RT did not significantly change the detrimental effects of LVI. Patients with LVI who underwent RT still had a poor DFS (Fig. 4A). Similarly, in the patients treated with RT
( $n=47)$, LVI did not reveal the negative prognostic effect on LRRFS ( $p=0.174 ; 3$-year LRRFS 96.7\% vs. 76.2\%). However, among the patients treated without RT ( $n=16)$, LVI had detrimental effect on LRRFS ( $p=0.046 ; 3$-year LRRFS $85.7 \%$ vs. $66.7 \%)$. 


\section{Discussion and Conclusion}

The impact of LVI on the prognosis of TNBC patients has been relatively under-evaluated $[7,10,12]$. $L V I$ is more commonly observed in patients with TNBC than in those without TNBC [13]. Agarwal et al. [13] revealed that a high incidence of LVI is observed in TNBC patients than in non-TNBC patients. According to their study [13], LVI was observed in 13.5\% of non-TNBC patients. In contrast, LVI was observed in $20.4 \%$ of TNBC patients. Although LVI is not included in the AJCC staging system, it may be a significant prognostic factor of breast cancer according to previous studies [1,12]. Pistelli et al. [12] exhibited that LVI was an independent prognostic factor for non-metastatic TNBC patients in terms of DFS. Therefore, in this study, we aimed to verify the effect of LVI on the prognosis of patients with TNBC. Our results suggested that the effect of LVI was significant in the prognosis of patients with TNBC.

LVI is a strong prognostic factor that significantly affects the treatment outcomes of TNBC. LVI was a poor prognostic factor for both DFS and OS based on both univariate and multivariate analyses in our study. In addition, Mohammed et al. [14] suggested that the development of distant metastasis was related to $L V I$, which is consistent with our results. In this study, distant metastasis mostly occurred in the patients with LVI. Although RT reduces the adverse effects of LVI on the prognosis of patients [15], the overall trend is still poor. The locoregional recurrence rate was slightly lower in patients with LVI who received RT than in those who did not receive RT. For several reasons, RT can decrease recurrence. Some genes that are associated with TNBC, particularly BRCA cancer genes, are known as radiosensitive tumors [9]. The decrease in the recurrence rate in patients with $\mathrm{LVI}$ is related to tumor cell killing via RT. However, microvascular contracture caused by RT may also be related to its effects. Microvascular contractures on tumor microenvironment that are associated with RT may contribute to the increased rate of DFS. Modified and intensified RT might be helpful in improving the prognosis of patients with LVI [16]. Moreover, the exclusion of RT in treatment of TNBC patients with LVI after BCS should not be considered in the clinical practice. In addition, the use of RT should be considered when LVI is observed after MRM. Truong et al. [17] showed that $21.2 \%$ of T1-2NO patients with LVI experienced locoregional recurrence after MRM. Their previous study [18] also showed reduced locoregional recurrence risk in LVI patients treated with PMRT (16.7\% vs. 9.1\%). Taken together, RT can be used to alleviate the risk of recurrence in those patients with LVI.

As in other cancers, p53 is known as a prognostic marker of TNBC [19]. LVI is commonly found in patients with p53 loss. Interestingly, the patients whose samples were p53 positive on immunostaining had better prognosis than those whose samples were not p53 positive, based on the results of this study. Although the clinical meaning of p53 is controversial, p53 expression is associated with the prognosis of patients with TNBC. In general, patients with TNBC had a high frequency of p53 mutation. Based on the previous studies by Chae et al. [20], p53 positivity was associated with poor survival outcomes of TNBC patients. In contrast, according to a recent report by Lehmann-Che et al. [21], p53 positivity was associated with a good treatment response in patients who were treated with modern dose-intense chemotherapy regimen, including epirubicin and cyclophosphamide. Our study also supports this result. In the present study, the majority of the patients were treated with chemotherapy, and a good prognosis was observed in p53-positive patients. In summary, since most of the patients were treated with chemotherapy in this study, p53 can be a predictive biomarker of chemotherapeutic response [22]. In addition, p53 positivity was related with a lower distant metastasis rate. Powell et al. [23] revealed that p53 loss was related to an increased rate of tumor metastasis, which is similar to the results of the present study.

Bevacizumab, which inhibits the neovascularization of tumors, may be useful in the treatment of TNBC patients with LVI. Sa-Nguanraksa et al. [24] previously reported that the vascular endothelial growth factor can influence both LVI and lymph nodal metastases. In this regard, an anti-angiogenesis drug, such as bevacizumab, might be effective in some patients with LVI $[25,26]$. The recent study by Miles et al. [27] showed an increased progression-free survival (PFS) of the docetaxeland bevacizumab-treated groups than those that were treated with docetaxel alone. Although PFS was not evaluated in the present study because of the small number of patients who were treated with bevacizumab, its effects on patients with LVI may be promising and should be further evaluated.

The direct mechanism of TNBC recurrence in patients with LVI must then be evaluated. Further experimental studies should be conducted to verify the cause of recurrence in patients with LVI. Genomic analysis might also be helpful in the investigation of the mechanism of recurrence. Based on a genetic analysis, Klahan et al. [28] recently showed that TNFSF11, IL6ST, and EPAS1 could play an important role in patients with LVI. 
This study has some limitations because it is a retrospective study that could be easily affected by selection bias. The sample size was relatively small. The detailed RT field was not specifically analyzed. The failure pattern might be affected by the degree of irradiation in the regional lymph nodal area. The study consisted of a fairly heterogeneous population of patients. Some of the patients underwent neoadjuvant chemotherapy, and a small number of patients did not receive $\mathrm{RT}$ after $\mathrm{BCS}$. All patients were classified according to pathologic staging, and patients with pN1-N3 were classified under the N-positive group. Thus, the significance of $\mathrm{N}$ stage on prognosis can be observed. Therefore, caution should be undertaken in giving clinical meaning based on the results of this study

In conclusion, LVI has a significant influence on the prognosis of TNBC patients after surgery. Although not perfect, adjuvant RT may reduce the negative prognostic effects of LVI. Therefore, further studies should be conducted to obtain an effective treatment strategy that can overcome the negative effects of LVI on prognosis. In addition, the development of a more efficient treatment method is needed.

\section{Conflict of Interest}

No potential conflict of interest relevant to this article was reported.

\section{References}

1. Rakha EA, Martin S, Lee AH, et al. The prognostic significance of lymphovascular invasion in invasive breast carcinoma. Cancer 2012;118:3670-80.

2. Liu $Y L$, Saraf $A$, Lee $S M$, et al. Lymphovascular invasion is an independent predictor of survival in breast cancer after neoadjuvant chemotherapy. Breast Cancer Res Treat 2016;157:555-64.

3. Schoppmann SF, Bayer G, Aumayr K, et al. Prognostic value of lymphangiogenesis and lymphovascular invasion in invasive breast cancer. Ann Surg 2004;240:306-12.

4. Krishnamurti U, Wetherilt CS, Yang J, Peng L, Li X. Tumorinfiltrating lymphocytes are significantly associated with better overall survival and disease-free survival in triplenegative but not estrogen receptor-positive breast cancers. Hum Pathol 2017;64:7-12.

5. Lee HJ, Park IA, Song IH, et al. Tertiary lymphoid structures: prognostic significance and relationship with tumourinfiltrating lymphocytes in triple-negative breast cancer. J Clin
Pathol 2016;69:422-30.

6. Shen $S$, Sun $Q$, Liang $Z$, et al. A prognostic model of triplenegative breast cancer based on miR-27b-3p and node status. PLoS One 2014;9:e100664.

7. Budakoglu B, Altundag K, Aksoy S, et al. Outcome of 561 nonmetastatic triple negative breast cancer patients: multi-center experience from Turkey. J BUON 2014;19:872-8.

8. Dai $X, L i T$, Bai $Z$, et al. Breast cancer intrinsic subtype classification, clinical use and future trends. Am J Cancer Res 2015:5:2929-43.

9. Wahba HA, El-Hadaad HA. Current approaches in treatment of triple-negative breast cancer. Cancer Biol Med 2015;12:10616.

10. Gujam FJ, Going JJ, Mohammed ZM, Orange C, Edwards J, McMillan DC. Immunohistochemical detection improves the prognostic value of lymphatic and blood vessel invasion in primary ductal breast cancer. BMC Cancer 2014;14:676.

11. Kim HA, Seong MK, Kim EK, et al. Evaluation of the survival benefit of different chemotherapy regimens in patients with T1-2N0 triple-negative breast cancer. J Breast Cancer 2015;18:271-8.

12. Pistelli M, Pagliacci $A$, Battelli N, et al. Prognostic factors in early-stage triple-negative breast cancer: lessons and limits from clinical practice. Anticancer Res 2013;33:2737-42.

13. Agarwal G, Nanda G, Lal $P$, et al. Outcomes of triple-negative breast cancers (TNBC) compared with non-TNBC: does the survival vary for all stages? World J Surg 2016;40:1362-72.

14. Mohammed ZM, McMillan DC, Edwards J, et al. The relationship between lymphovascular invasion and angiogenesis, hormone receptors, cell proliferation and survival in patients with primary operable invasive ductal breast cancer. BMC Clin Pathol 2013;13:31.

15. Abdulkarim BS, Cuartero J, Hanson J, Deschenes J, Lesniak D, Sabri S. Increased risk of locoregional recurrence for women with T1-2No triple-negative breast cancer treated with modified radical mastectomy without adjuvant radiation therapy compared with breast-conserving therapy. J Clin Oncol 2011;29:2852-8.

16. Orecchia R. Tailoring radiotherapy according to cancer subtypes. Breast 2017;34 Suppl 1:S91-S94.

17. Truong PT, Lesperance $M$, Culhaci $A$, Kader HA, Speers $\mathrm{CH}_{\text {, }}$ Olivotto IA. Patient subsets with T1-T2, node-negative breast cancer at high locoregional recurrence risk after mastectomy. Int J Radiat Oncol Biol Phys 2005;62:175-82.

18. Truong PT, Olivotto IA, Speers CH, Wai ES, Berthelet $E$, Kader HA. A positive margin is not always an indication for radiotherapy after mastectomy in early breast cancer. Int J 
Radiat Oncol Biol Phys 2004;58:797-804.

19. Rakha EA, El-Sayed ME, Green AR, Lee AH, Robertson JF, Ellis I0. Prognostic markers in triple-negative breast cancer. Cancer 2007;109:25-32.

20. Chae BJ, Bae JS, Lee $A$, et al. p53 as a specific prognostic factor in triple-negative breast cancer. Jpn J Clin Oncol 2009;39:21724.

21. Lehmann-Che J, Andre F, Desmedt C, et al. Cyclophosphamide dose intensification may circumvent anthracycline resistance of p53 mutant breast cancers. Oncologist 2010;15:246-52.

22. Jin J, Zhang W, Ji W, Yang F, Guan X. Predictive biomarkers for triple negative breast cancer treated with platinum-based chemotherapy. Cancer Biol Ther 2017;18:369-78.

23. Powell E, Piwnica-Worms D, Piwnica-Worms H. Contribution of p53 to metastasis. Cancer Discov 2014;4:405-14.

24. Sa-Nguanraksa D, Chuangsuwanich T, Pongpruttipan $T_{1}$ O-Charoenrat P. High vascular endothelial growth factor gene expression predicts poor outcome in patients with nonluminal A breast cancer. Mol Clin Oncol 2015;3:1103-8.
25. Cameron D, Brown J, Dent R, et al. Adjuvant bevacizumabcontaining therapy in triple-negative breast cancer (BEATRICE): primary results of a randomised, phase 3 trial. Lancet Oncol 2013;14:933-42.

26. Ma $X$, Wang $X$, Huang J, et al. Bevacizumab addition in neoadjuvant treatment increases the pathological complete response rates in patients with HER-2 negative breast cancer especially triple negative breast cancer: a meta-analysis. PLoS One 2016;11:e0160148.

27. Miles DW, Chan A, Dirix LY, et al. Phase III study of bevacizumab plus docetaxel compared with placebo plus docetaxel for the first-line treatment of human epidermal growth factor receptor 2-negative metastatic breast cancer. $J$ Clin Oncol 2010;28:3239-47.

28. Klahan S, Wong HS, Tu SH, et al. Identification of genes and pathways related to lymphovascular invasion in breast cancer patients: a bioinformatics analysis of gene expression profiles. Tumour Biol 2017 Jun 27 [Epub]. http://doi. org/10.1177/1010428317705573. 